Check for updates

Cite this: Chem. Commun., 2022, 58,815

Received 30th September 2021

Accepted 6th December 2021

DOI: $10.1039 / \mathrm{d} 1 \mathrm{cc} 05520 \mathrm{~h}$

rsc.li/chemcomm

\title{
The rise of an exciton in solid ammonia
}

\author{
Andrew Cassidy, (D)*a Rachel L. James, (D) ${ }^{b}$ Anita Dawes (D) ${ }^{* c}$ and David Field (D) ${ }^{d}$
}

\begin{abstract}
We trace a polymorphic phase change in solid ammonia films through the emergence of a Frenkel exciton at $194.4 \mathrm{~nm}$, for deposition temperatures of $48 \mathrm{~K}, 50 \mathrm{~K}$ and $52 \mathrm{~K}$. Observations on a timescale of hours give unparalleled access to the individual processes of nucleation and the phase change itself. The excitonic transition is forbidden in the low temperature phase, but greater flexing of the solid state structure in the higher temperature phase makes the transition allowed, as the nano-crystals approach $\sim 30$ unit cells through nucleation. We find activation energies of $21.7 \pm 0.6 \mathrm{~kJ} \mathrm{~mol}^{-1}$ for nucleation and $22.8 \pm 0.6 \mathrm{~kJ} \mathrm{~mol}^{-1}$ for the phase change, corresponding to the breaking of two to three hydrogen bonds.
\end{abstract}

Solid state phase transformations, or polymorphic phase transitions, ${ }^{1-3}$ are encountered in an array of fields ranging from drug manufacture, ${ }^{4}$ electronics, ${ }^{5}$ chocolate production ${ }^{6}$ to the interstellar medium. ${ }^{7}$ Polymorphic transitions can be described by two consecutive and distinct processes, the nucleation phase in the parent matrix and the growth of these nuclei to complete the transformation. Despite their broad importance, the processes occurring during nucleation and recrystallization at the molecular scale have proved largely inaccessible. We show here how vacuum ultraviolet (VUV) absorption spectra, recorded for thin cryo-films of ammonia, reveal separately the nucleation rate for crystallite formation in solid ammonia in real time and the growth rate of these nuclei, until the phase conversion nears completion.

We study films a few nanometres thick, deposited by physical vapour deposition under ultra-high vacuum (UHV). It is known from earlier work that such films form in only two crystalline phases, ${ }^{8-10}$ displaying a polymorphic phase transition at a deposition temperature, $T_{\mathrm{d}}$, of $\sim 50 \mathrm{~K}$. These phases are referred to below as phase II,

\footnotetext{
${ }^{a}$ Center for Interstellar Catalysis and Department of Physics and Astronomy, Aarhus University, Denmark.E-mail:amc@phys.au.dk

${ }^{b}$ Department of Earth and Environmental Sciences, University of Manchester, UK

${ }^{c}$ School of Physical Sciences, The Open University, UK.

E-mail: anita.dawes@open.ac.uk

${ }^{d}$ Department of Physics and Astronomy, Aarhus University, Denmark
}

for low $T_{\mathrm{d}}$ and phase I, for high $T_{\mathrm{d}}{ }^{11}$ The ammonia moieties lie on a cubic unit cell in the space group P213- $\mathrm{T}^{4}$, which enables hydrogen bonding. ${ }^{12,13}$ Films belong to the family of 'spontelectric' solids, ${ }^{14}$ displaying spontaneously generated electric fields created on deposition, so-called 'spontelectric fields'. Phases I and II differ in their spontelectric properties. ${ }^{8}$ Fields arise through dipole orientation, which results in a bound polarization charge at the surface/vacuum interface of the film. In phase II, the average angle of ammonia moieties to the horizontal varies between $1.79^{\circ}$ at $T_{\mathrm{d}}=20 \mathrm{~K}$ and $1.58^{\circ}$ at $50 \mathrm{~K}$. In phase I, values are $0.54^{\circ}$ at $55 \mathrm{~K}$ falling to $0.27^{\circ}$ at $80 \mathrm{~K},{ }^{8}$ demonstrating subtle differences in average orientation in the structures of phase I and II.

VUV absorption spectra of ammonia ices condensed between $48 \mathrm{~K}$ and $52 \mathrm{~K}$ were observed to change with time, most prominently via the emergence of a sharp peak at $194.4 \mathrm{~nm}$, which we attribute to the emergence of a Frenkel exciton. Rate of emergence of the exciton peak is associated with a polymorphic transition between phase II and phase I, gives the rate of the phase change and the delayed emergence of the peak gives the nucleation rate.

General features of VUV absorption spectra for thin films of ammonia are shown in Fig. 1. Curve a displays the absorption spectrum for a film with $T_{\mathrm{d}}=20 \mathrm{~K}$, phase II, and curve b with $T_{\mathrm{d}}=65 \mathrm{~K}$, phase I. The sharp absorption peak around $194.4 \mathrm{~nm}$ (curve b) can be observed, immediately following deposition, only in films prepared with $T_{\mathrm{d}} \geq 55 \mathrm{~K}$. It forms after minutes or hours at lower temperatures and it is altogether absent below $48 \mathrm{~K}$. Curve c in Fig. 1, was recorded through deposition at $20 \mathrm{~K}$ and subsequent anneal to $65 \mathrm{~K}$. There is a shoulder evident at $194.4 \mathrm{~nm}$.

Absorption lines are associated with transitions involving excitation of the $\tilde{\mathrm{X}}^{1} \mathrm{~A}_{1}$ ground state of $\mathrm{NH}_{3}$ to Rydberg states, well characterised in gas phase spectra. ${ }^{15,16}$ The Rydberg states of $\mathrm{NH}_{3}$ in the gas phase are planar and of $D_{3 \mathrm{~h}}$ symmetry, whereas the ground state is pyramidal, belonging to $C_{3 \mathrm{v}}$. We propose here that in the low temperature phase II, excited states of $\mathrm{NH}_{3}$ remain $C_{3 \mathrm{v}}$, whereas only in phase I do they become $D_{3 \mathrm{~h}}$. The $\tilde{\mathrm{A}} \leftarrow \tilde{\mathrm{X}}$ transition has a maximum at 


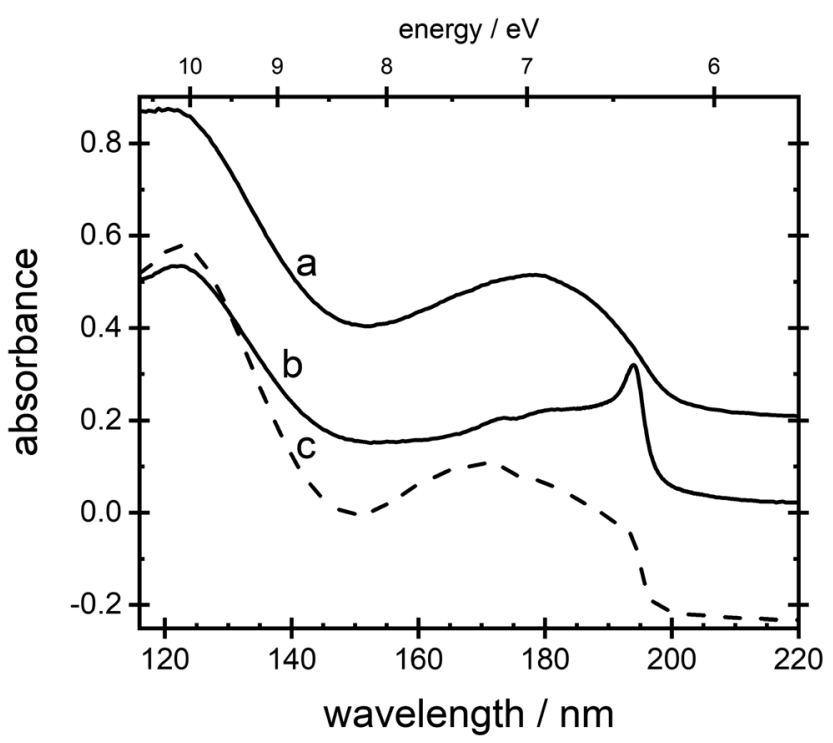

Fig. 1 VUV absorption spectrum for a thin film of ammonia prepared with a $T_{\mathrm{d}}=20 \mathrm{~K}, \mathrm{~b} T_{\mathrm{d}}=65 \mathrm{~K}$ and $\mathrm{c} T_{\mathrm{d}}=20 \mathrm{~K}$ and annealed to $65 \mathrm{~K}$ showing a shoulder around $194.4 \mathrm{~nm}$. Spectra a and $c$ have been offset in absorbance for clarity.

$193.85 \mathrm{~nm}$ in gas phase spectra, in the $\nu_{2}$ umbrella mode progression. Thus in phase I, the $194.4 \mathrm{~nm}$ absorption line may be attributed to the $\tilde{\mathrm{A}}^{1} \mathrm{~A}_{2}^{\prime \prime} \leftarrow \tilde{\mathrm{X}}^{1} \mathrm{~A}_{1}^{\prime}$ transition, with the molecules able to flex inter-molecular hydrogen bonds ${ }^{12}$ and tunnel through the planar $D_{3 h}$ structure in the $\tilde{X}$ state.

The wavelength of the $\tilde{\mathrm{A}}^{1} \mathrm{~A}_{2}^{\prime \prime} \leftarrow \tilde{\mathrm{X}}^{1} \mathrm{~A}_{1}^{\prime}$ transition is independent of $T_{\mathrm{d}}$ over the range of temperature between $48 \mathrm{~K}$ and $80 \mathrm{~K}$, and thus of changes in the $T_{\mathrm{d}}$-dependent spontelectric field in the film. ${ }^{8}$ The $194.4 \mathrm{~nm}$ transition therefore involves a Frenkel exciton, in which the hole and electron reside on the same molecular species and the absorption wavelength is insensitive to the electric field, in contrast to Wannier-Mott excitons. ${ }^{8}$

In the low temperature phase II, we suggest that $\mathrm{NH}_{3}$ has a vanishingly small probability of assuming $D_{3 \mathrm{~h}}$ symmetry. This arises through inhibition of the $\nu_{2}$ umbrella motion, which we attribute to a significantly higher barrier to inversion in phase II, than in phase I. This results in a negligible amplitude in the $\tilde{\mathrm{A}}^{1} \mathrm{~A}_{2}^{\prime \prime} \leftarrow \tilde{\mathrm{X}}^{1} \mathrm{~A}_{1}^{\prime}$ transition. A tunnelling frequency of $1 \mathrm{MHz}$ was reported for solid $\mathrm{ND}_{3}$ at $2 \mathrm{~K}^{17}$ The gas phase tunnelling frequency is $1.6 \mathrm{GHz}$ for $\mathrm{ND}_{3},{ }^{18}$ where this factor of $>10^{3}$, between the gas phase and the solid state, illustrates the influence of a barrier to inversion in phase II, as suggested, albeit for the deuterated species. If the ground state remains $\tilde{\mathrm{X}}^{1} \mathrm{~A}_{1}$, that is, effectively lacking umbrella motion, and the $\tilde{\mathrm{A}}$ state also remains in $C_{3 \mathrm{v}}$ as proposed above, then the $\tilde{\mathrm{A}} \leftarrow \tilde{\mathrm{X}}$ is forbidden and the $194.4 \mathrm{~nm}$ line is absent in phase II, as observed.

Spectra of phases I and II remain virtually indistinguishable between $145 \mathrm{~nm}$ and $115 \mathrm{~nm}$, curves a and b, Fig. 1, involving $\tilde{\mathrm{D}}, \tilde{\mathrm{E}}, \tilde{\mathrm{F}}$ and $\tilde{\mathrm{G}} \leftarrow \tilde{\mathrm{X}}$ transitions. This is consistent with symmetry considerations which show that these transitions are all allowed, whether the excited states are of $C_{3 \mathrm{v}}$ or $D_{3 \mathrm{~h}}$ symmetry.

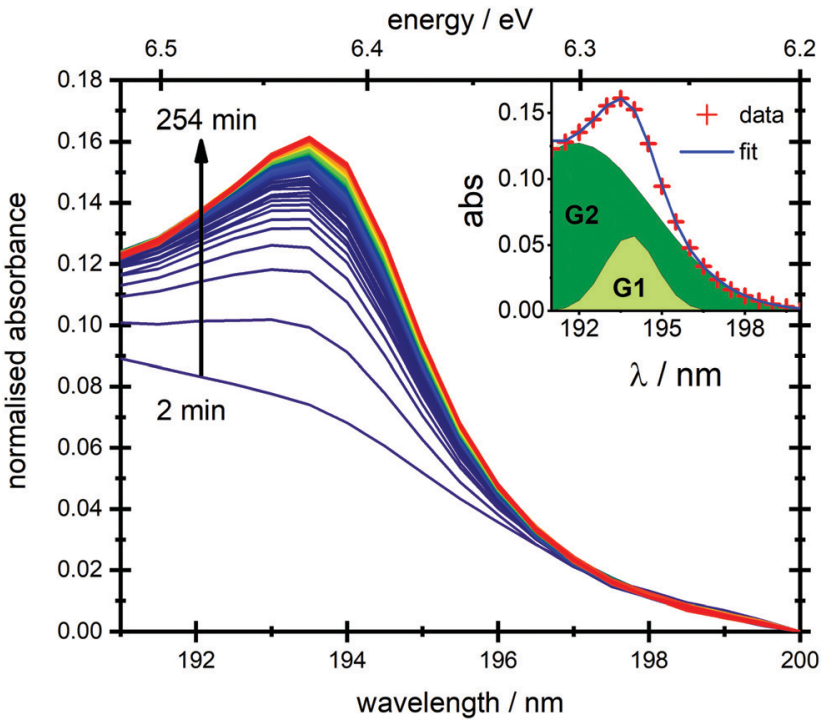

Fig. 2 VUV absorption spectra for a $6 \mathrm{~nm}$ thick ammonia film deposited at $52 \mathrm{~K}$. Spectra were recorded as a function of time, starting at effective time zero, ${ }^{19}$ moving from blue to red at later times. All spectra are backgroundsubtracted such that absorbance at $200 \mathrm{~nm}$ is set to zero. The inset shows a 2-Gaussian peak fit to the final absorption spectrum, obtained after 4 hours, with G1 representing the peak at 194.4 nm, and G2 representing the background.

An example of the observed growth with time of the $194.4 \mathrm{~nm}$ peak, and the rise of the exciton for $T_{\mathrm{d}}=52 \mathrm{~K}$, is presented in Fig. 2. Data in Fig. 2 were collected over a period of 4 hours and demonstrate the absence of any absorption peak at $194.4 \mathrm{~nm}$ at the earliest times, ${ }^{19}$ followed by the growth of the peak over this time period. Each individual spectrum in Fig. 2 was fit with two Gaussians to extract the change in amplitude of the peak at $194.4 \mathrm{~nm}$ as a function of time. As an example, Gaussian fits to the final spectrum, when the peak is fully developed at long time, are presented as an inset to Fig. 2.

The change in amplitude of the peak at $194.4 \mathrm{~nm}$, labelled G1 in Fig. 2, is shown as a function of time, for 3 different films prepared with $T_{\mathrm{d}}=48 \mathrm{~K}, 50 \mathrm{~K}$ and $52 \mathrm{~K}$, in Fig. 3. Changing $T_{\mathrm{d}}$ has three notable effects on the shape of the growth curves in Fig. 3. First, we note the variation with $T_{\mathrm{d}}$ of the maximum absorbance, $A_{\max }$. This quantity is higher for higher $T_{\mathrm{d}}$, with $A_{\max }=0.14$ for $52 \mathrm{~K}, A_{\max }=0.11$ for $50 \mathrm{~K}$ and $A_{\max }=0.015$ for $T_{\mathrm{d}}=48 \mathrm{~K}$. Second, there is a time offset, $t_{\text {offset }}$, before the peak begins to grow for data with $T_{\mathrm{d}}=48 \mathrm{~K}$, a shorter offset with $T_{\mathrm{d}}=50 \mathrm{~K}$, and the peak is already vestigially present for the shortest measurable times of 120 seconds for $T_{\mathrm{d}}=52 \mathrm{~K}$. Third, the rate at which the peak grows is strongly temperature dependent. Table 1 collates these results. Additional data collected at $T_{\mathrm{d}}=50 \mathrm{~K}$, for $4.2 \mathrm{~nm}$ and $8.4 \mathrm{~nm}$ films, show that the rate of peak growth is independent of film thickness. From this we conclude that phase change in our thin films occurs via bulk processes and is not significantly influenced by the presence of interfaces.

The polymorphic recrystallization from phase II to phase I may be seen to involve two distinct stages. The first stage is 


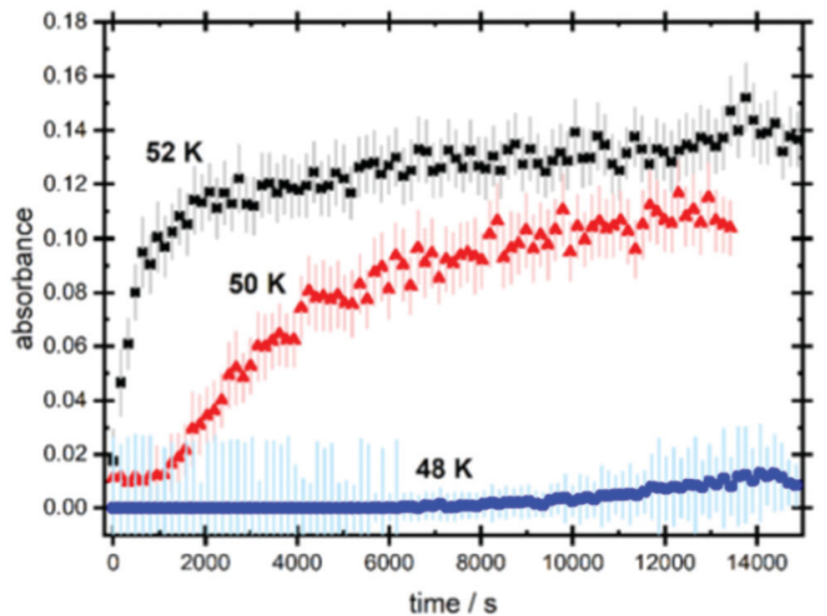

Fig. 3 The change in amplitude of the $\tilde{\mathrm{A}}^{1} \mathrm{~A}_{2}^{\prime \prime} \leftarrow \tilde{\mathrm{X}}^{1} \mathrm{~A}_{1}^{\prime}$ exciton peak at $194.4 \mathrm{~nm}$ as a function of time, for ammonia films of thickness $6 \mathrm{~nm}$ prepared at three different $T_{\mathrm{d}}$. Data are shown as points and error bars as light lines. Errors result from the error in the Gaussian fits presented in Fig. 2.

maintained for the offset times in Table 1. This stage is associated with fluctuations leading to the accumulation of nuclei, that is, local structures tending to a phase 1 structure, which once formed, fail to survive and decay back towards their phase II conformation. These metastable nuclei decay because they are generated in an energetically unfavourable environment. In the experiment, the observation of this stage is consistent with the absence of any absorption in the $\tilde{\mathrm{A}}^{1} \mathrm{~A}_{2}^{\prime \prime} \leftarrow \tilde{\mathrm{X}}^{1} \mathrm{~A}_{1}^{\prime}$ excitonic transition for the period $t_{\text {offset }}$. This transition is indicative of the presence of phase I.

The system thus remains quiescent for $t_{\text {offset }}$ but then, through some fluctuation, finds sufficient extent of material in a crystallite for the excitonic transition to take place, via umbrella motion. These may then give rise to zones of critical size and the formation of critical nuclei, which grow rather than decay. Such nuclei conform more to the phase I than the phase II environment and become the seeds of the new and larger crystallites of phase I, yielding the $194.4 \mathrm{~nm}$ absorption. New here is that we reveal the unsuccessful attempts to form phase I structures of sufficient conformity to survive within this new phase.

Following classical nucleation theory, we consider a rate, $J$, for the formation of nuclei of the target phase I, that will grow instead of shrink, on forming from nuclei of phase II. This rate is given by an Arrhenius activated process with a Gibbs free-energy barrier, $\Delta G_{\text {nucl }}^{*} ;^{20,21}$

$$
J=J_{0}\left(\mathrm{e}^{\frac{-\Delta G_{\text {nucl }}^{*}}{k T}}\right) \cdot(\varepsilon l)^{-1}
$$

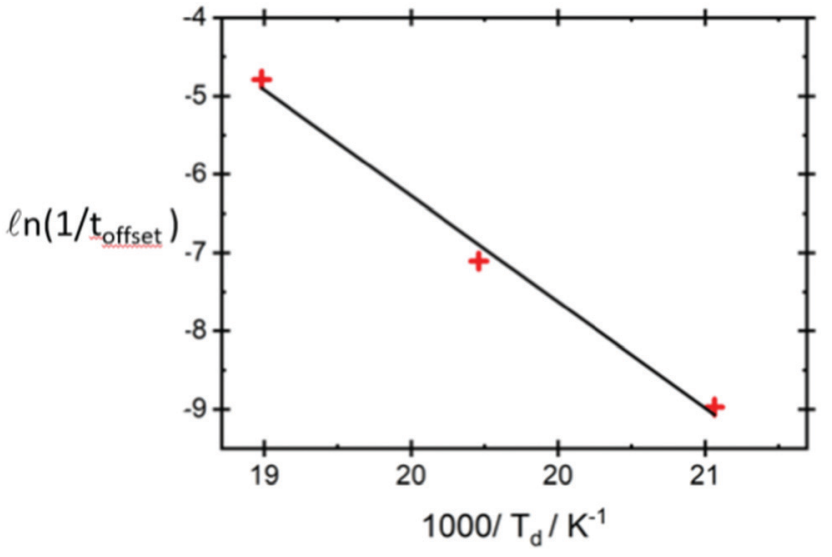

Fig. 4 An Arrhenius analysis, based upon eqn (1), using values of $t_{\text {offset, }}$ Table 1, yielding $\Delta G_{\text {nucl }}^{*}$, of $21.7 \pm 0.6 \mathrm{~kJ} \mathrm{~mol}^{-1}$; see eqn (1).

where $k$ is Boltzmann's constant, $T$ is temperature, and $\varepsilon l$ represents the absorption coefficient of solid $\mathrm{NH}_{3}$ times the film thickness, with units of volume (see caption to Table 1). We take this effective rate for nucleation to be proportional to the inverse of $t_{\text {offset }}$. The ability to explore configurations may therefore be expressed as $1 / t_{\text {offset }} \propto \exp \left(-\Delta G_{\text {nucl }}^{*} / k T\right)$. Application of this expression is shown in Fig. 4. This yields a barrier to attainment of a critical configuration, $\Delta G_{\text {nucl }}^{*}$, of $21.7 \pm 0.6 \mathrm{~kJ} \mathrm{~mol}^{-1}$. The activation energy barrier, $\Delta G_{\text {cryst }}^{*}$, of the re-crystallization process itself, may be determined from the initial rates of $194.4 \mathrm{~nm}$ peak growth, in Table 1, column 3, for $T_{\mathrm{d}}=48 \mathrm{~K}, 50 \mathrm{~K}$ and $52 \mathrm{~K}$. An Arrhenius analysis gives a free energy barrier of $22.8 \pm 0.6 \mathrm{~kJ} \mathrm{~mol}^{-1}$, within the errors the same value as that for nucleation.

With regard to the values of $\Delta G_{\text {nucl }}^{*}$ and $\Delta G_{\text {cryst }}^{*}$, the strength of the hydrogen bond in ammonia has been calculated to be $9.3 \mathrm{~kJ} \mathrm{~mol}^{-1}$. $^{22}$ The values of $\Delta G_{\text {nucl }}^{*}$ and $\Delta G_{\text {cryst }}^{*}$ therefore suggest the breaking of two to three hydrogen bonds to form the transition state, in both nucleation and crystallization stages of the phase change. The result that $\Delta G_{\text {nucl }}^{*} \approx \Delta G_{\text {cryst }}^{*}$ reflects the fact that the physical processes for nucleation and crystallization are very comparable, requiring similar rupture of the lattice structure. We note that the activation energy associated with the phase change falls below the energy required for molecular desorption, measured to be $29 \mathrm{~kJ} \mathrm{~mol}^{-1}$ and calculated to be $31.4 \mathrm{~kJ} \mathrm{~mol}^{-1}$. ${ }^{23}$

We have shown in previous work that in phase I, crystallites formed at $65 \mathrm{~K}$ contain 422 unit cells, at $60 \mathrm{~K}, 189$ unit cells and at $55 \mathrm{~K}, 59$ unit cells. $^{8}$ Extrapolation of these figures suggests $34 \pm 8$ at $52 \mathrm{~K}, 20 \pm 4$ at $50 \mathrm{~K}$ and $10 \pm 2$ unit cells at $48 \mathrm{~K}(1 \sigma)$. Thus crystallites may contain on average between $2 \times 2 \times 2$ and

Table 1 Parameters describing the change in amplitude of the absorption peak at $194.4 \mathrm{~nm}$ in $6 \mathrm{~nm}$ films of ammonia, deposited at various $T_{\mathrm{d}}$. Values are peculiar to $6 \mathrm{~nm}$ films, since data derive from absorbance, effectively containing units of volume

\begin{tabular}{llll}
\hline$T_{\mathrm{d}} / \mathrm{K}$ & $t_{\text {offset }} / \mathrm{s}^{19}$ & Initial rate of peak growth/absorbance $/ \mathrm{s}^{-1}$ & Rate of growth of nuclei/s \\
\hline 48 & $7870 \pm 350$ & $1.49 \times 10^{-6} \pm 8 \times 10^{-8}$ & $1.27 \times 10^{-4} \pm 6 \times 10^{-6}$ \\
50 & $1220 \pm 44$ & $1.95 \times 10^{-5} \pm 8 \times 10^{-7}$ & $8.2 \times 10^{-4} \pm 3 \times 10^{-5}$ \\
52 & $120 \pm 8$ & $1.19 \times 10^{-4} \pm 6 \times 10^{-6}$ & $8.33 \times 10^{-3} \pm 6 \times 10^{-4}$
\end{tabular}


$3 \times 2 \times 2$ unit cells, at $48 \mathrm{~K}, 2 \times 3 \times 3$ to $3 \times 3 \times 3$ unit cells at $50 \mathrm{~K}$, and $3 \times 3 \times 3$ to $3 \times 4 \times 4$ unit cells at $52 \mathrm{~K}$. Results in Fig. 3 show that the maximum absorbance, after $14000 \mathrm{~s}$, drops markedly with $T_{\mathrm{d}}$ between $52 \mathrm{~K}$ and $48 \mathrm{~K}$, in parallel with crystallite size. We postulate that there is some minimum crystallite size which gives sufficient elbow room for the hydrogen bonds to flex and enable the $\tilde{\mathrm{A}}^{1} \mathrm{~A}_{2}^{\prime \prime} \leftarrow \tilde{\mathrm{X}}^{1} \mathrm{~A}_{1}^{\prime}$ excitonic transition. For any $T_{\mathrm{d}}$, there is a distribution of crystallite sizes and the proportion of crystallites with some necessary minimum size is lower for lower $T_{\mathrm{d}}$. We suggest that the average size of crystallites at $48 \mathrm{~K}$, with 8 to 12 unit cells, is too small for sufficient flexing to allow the excitonic transition. Therefore only the tail of the distribution of sizes is sufficient to support this transition. At higher $T_{\mathrm{d}}$, crystallites progress to $2 \times 3 \times 3$ to $3 \times 3 \times$ 3 unit cells at $50 \mathrm{~K}$ and $3 \times 3 \times 3$ to $3 \times 4 \times 4$ at $52 \mathrm{~K}$, with an increasingly higher proportion able to support the $\tilde{\mathrm{A}}^{1} \mathrm{~A}_{2}^{\prime \prime} \leftarrow \tilde{\mathrm{X}}^{1} \mathrm{~A}_{1}^{\prime}$ excitonic transition.

The crystal structure of ammonia can be considered as an intermediate step on the scale between the H-bond-dominated structure of water ice and the quantum cryo-crystals of methane, ${ }^{24}$ the latter lacking any hydrogen bonding. It is likely that the phenomenon, reported here for solid ammonia, could be transferred to these other species in an effort to compare the dynamics of crystallisation. Water, as a polar molecule, would also be a good candidate in which to pursue the spontelectric effect and the nature of phase changes, noting the contribution that studies of the spontelectric effect in ammonia have made to our understanding of the current system. Polarisation behaviour in water ices has been reported elsewhere $e^{25,26}$ but temperature dependent VUV absorption data for water-ice films are not available.

AC thanks the Danish National Research Foundation (Center for Interstellar Catalysis, DNRF150). RJ acknowledges the STFC for her PhD Studentship under grant no. ST/N50421X/1. The research leading to this result has been supported by the project CALIPSOplus under the Grant Agreement 730872 from the EU Framework Programme for Research and Innovation HORIZON 2020. We acknowledge the Centre for Storage Ring Facilities and staff at Aarhus (ISA) for granting access to the AUUV beamline on the ASTRID2 storage ring with special thanks to Nykola Jones.

\section{Conflicts of interest}

There are no conflicts of interest to declare.

\section{Notes and references}

1 J. D. Dunitz, Pure Appl. Chem., 1991, 63, 177-185.

2 J. Anwar and D. Zahn, Adv. Drug Delivery Rev., 2017, 117, 47-70.

3 S. Karthika, T. K. Radhakrishnan and P. Kalaichelvi, Cryst. Growth Des., 2016, 16, 6663-6681.

4 S. Datta and D. J. W. Grant, Nat. Rev. Drug Discovery, 2004, 3, 42-57. 5 P. Yu, Y. Zhen, H. Dong and W. Hu, Chem, 2019, 5(11), 2814-2853.

6 R. L. Wille and E. S. Lutton, J. Am. Oil Chem. Soc., 1966, 43, 491-496.

7 K. I. Öberg, A. C. A. Boogert, K. M. Pontoppidan, S. van den Broek, E. F. van Dishoeck, S. Bottinelli, G. A. Blake and N. J. Evans, ApJ, 2011, 740, 109.

8 A. Cassidy, R. L. James, A. Dawes and D. Field, ChemistryOpen, 2020, 9, 983-990.

9 A. Dawes, R. J. Mukerji, M. P. Davis, P. D. Holtom, S. M. Webb, B. Sivaraman, S. V. Hoffmann, D. A. Shaw and N. J. Mason, J. Chem. Phys., 2007, 126, 244711.

10 W. Zheng and R. I. Kaiser, Chem. Phys. Lett., 2007, 440, 229-234.

11 The literature has labelled a phase of solid ammonia stable at pressures above $5.1 \mathrm{kbar}$ as phase II. There is no evidence that the low $\mathrm{T}_{\mathrm{d}}$ phase II in the present manuscript forms in these high pressure environments. Our phase II bears no relation to this high pressure phase save that it is another stable phase of crystalline ammonia. For details on the phase diagram for ammonia at high pressures we refer the reader to: L. Huang, Y. Han, J. Liu, X. He and J. Li, Sci. Rep., 2020, 10, 7546.

12 I. Olovsson and D. H. Templeton, Acta Crystallogr., 1959, 12, 827-832.

13 R. Boese, N. Niederprüm, D. Bläser, A. Maulitz, M. Y. Antipin and P. R. Mallinson, J. Phys. Chem. B, 1997, 101, 5794-5799.

14 D. Field, O. Plekan, A. Cassidy, R. Balog, N. C. Jones and J. Dunger, Int. Rev. Phys. Chem., 2013, 32, 345-392.

15 S. Leach, H.-W. Jochims and H. Baumgärtel, Phys. Chem. Chem. Phys., 2005, 7, 900-911.

16 P. Limão-Vieira, N. C. Jones, S. V. Hoffmann, D. Duflot, M. Mendes, A. I. Lozano, F. Ferreira da Silva, G. García, M. Hoshino and H. Tanaka, J. Chem. Phys., 2019, 151, 184302.

17 A. W. Hewat and C. Riekel, Acta Crystallogr., Sect. A: Cryst. Phys., Diffr., Theor. Gen. Crystallogr., 1979, 35, 569-571.

$18 \mathrm{~J}$. van Wijngaarden and W. Jäger, J. Chem. Phys., 2001, 114, 3968-3976.

19 A 120s delay occurs between the completion of film dosing and the collection of the first spectrum. Time zero represents the recording of the first spectrum.

20 K. A. Jackson, Kinetic Processes: Crystal Growth, Diffusion, and Phase Transitions in Materials, John Wiley \& Sons, 2010.

21 J. W. P. Schmelzer, J. Non-Cryst. Solids, 2010, 356, 2901-2907.

22 C. A. Morrison and M. M. Siddick, Chem. - Eur. J., 2003, 9, 628-634.

23 L. L. Shipman, A. W. Burgess and H. A. Scheraga, J. Phys. Chem., 1976, 80, 52-54.

24 A. Leont'eva, A. Prokhorov, V. Romanusha and V. Efimov, Low Temp. Phys., 2019, 45, 260-267.

25 M. J. Iedema, M. J. Dresser, D. L. Doering, J. B. Rowland, W. P. Hess, A. A. Tsekouras and J. P. Cowin, J. Phys. Chem. B, 1998, 102, 9203-9214.

26 C. Bu, J. Shi, U. Raut, E. H. Mitchell and R. A. Baragiola, J. Chem. Phys., 2015, 142, 134702. 